interpreted. Occasionally an actually misleading statement occurs, as when on page 116 in discussing the essential difference between the Cauchy-Kowalewski existence theorem for Laplace's equation and the "problem of Dirichlet" it is at least strongly implied that the former theorem does not apply to closed curves, whereas it applies exactly as well to closed curves as to open ones, but in both cases, and this is the essential point, to only a small neighborhood of the curve.

Judiciously used by a person who is able to perceive that he does not understand a thing when that is the actual case, the book will prove a source of inspiration.

\title{
MaXime Bôcher.
}

Analytische Geometrie auf der Kugel. Von Dr. RICHARD Heger. Sammlung Schubert, LIV. Leipzig, G. T. Göschen, $1908.12 \mathrm{mo}$. vii $+152 \mathrm{pp}$.

There is a spherical trigonometry ; why not also a spherical analytical geometry? This question interested mathematicians towards the end of the eighteenth century and the beginning of the nineteenth, and there resulted numerous papers published in the periodicals of the time. Certain problems had been solved at an earlier date and others have appeared up to within a decade ago. It is the object of the author of the little volume before us to bring this material together in a convenient form and to arrange it along the lines of the usual text upon plane analytic geometry. The book begins by explaining several coordinate systems upon the sphere. Dr. Heger adopts that one in which the homogeneous coordinates of a point are the sines of the angles whose arcs are drawn perpendicularly from the point to the sides of the spherical triangle of reference. This triangle of reference is assumed to be trirectangular. The homogeneous coordinates of a great circle are taken to be the point coordinates of one of its poles. Many of the formulas are exactly the same as the analogous formulas in plane geometry. For instance, the necessary and sufficient condition that a point $(x, y, z)$ lie upon a great circle $(u, v, w)$ is

$$
u x+v y+w z=0 .
$$

Small circles and conics in general are represented by quadratic equations. There is a theory of poles and polars and of tangents, all of which is analogous to plane analytic geometry. 
The book closes with a section devoted to spherical cubics. There are 152 pages, a bibliography, and a short index.

The book will find its greatest use in technical schools. Spherical trigonometry has come to be counted as one of the technical studies and taught in connection with geodetic surveying or with astronomy. It is the same with spherical analytic geometry from Dr. Heger's point of view, and the field of application is narrower.

Geometry upon the sphere is most interesting when studied as a correspondence between the sphere and some other surface - in particular, the plane. This point of view is hinted at in section 8 in explaining Gudermann's axial coordinates, but no general theory of correspondence between sphere and plane is worked out.

Dr. Heger's analytical geometry amounts to a correspondence between the sphere and the projective plane. There is another geometry upon the sphere arising from a one to one correspondence with the plane whose results are quite as useful, in their way, but which does not come within Dr. Heger's field of view.

\section{Wayland Dowling.}

Die Elemente der Mathematik. Von Émile Borel, Professor an der Sorbonne zu Paris. Vom Verfasser genehmigte deutsche Ausgabe, besorgt von Paul Stäckel, Professor zu Karlsruhe i. B. Erster Band: Arithmetik und Algebra. Mit 57 Textfiguren und 3 Tafeln. Leipzig und Berlin, B. G. Teubner, 1908. xvi +431 pp.

This work is a German translation, or rather a "Bearbeitung," in one volume, of the three French booklets published by Borel in 1903. Borel traverses in his texts the ground to be covered in arithmetic and algebra by pupils between the ages of 14 and 17, in accordance with the courses of study laid out officially in 1902. The distinctive feature of this movement lies in the emphasis laid on graphic work, on the concept of a variable and of a function. Stäckel says in his preface to the German edition that, in view of the wide divergence of opinion as to what can be accomplished in this line with elementary pupils, the only way of arriving at an understanding and thereby at an actual realization of the contemplated reform, appears to be in showing by an example just what that reform really aims to achieve and how the subject can be developed 